\title{
Methodological Guidelines Designed to Improve the Quality of Research on Cross-Country Skiing
}

\author{
Barbara Pellegrini $^{1,2} \cdot \emptyset y v i n d$ Sandbakk ${ }^{3} \cdot$ Thomas Stöggl $^{4} \cdot$ Matej Supej $^{5} \cdot$ Niels $_{\text {Ortenblad }}{ }^{6} \cdot$ Axel Schürer $^{7}$. \\ Thomas Steiner $^{8} \cdot$ Angelica Lunina $^{9} \cdot$ Chris Manhard $^{10} \cdot$ Hui Liu $^{11} \cdot$ Olli Ohtonen $^{12}$. Chiara Zoppirolli ${ }^{1,2}$. \\ Hans-Christer Holmberg ${ }^{10,11,13}$
}

Received: 16 December 2020 / Accepted: 26 January 2021 / Published online: 29 June 2021

(c) The Author(s) 2021

\begin{abstract}
Cross-country (XC) ski races involve a variety of formats, two different techniques and tracks with highly variable topography and environmental conditions. In addition, $\mathrm{XC}$ skiing is a major component of both Nordic combined and biathlon competitions. Research in this area, both in the laboratory and field, encounters certain difficulties that may reduce the reliability and validity of the data obtained, as well as complicate comparisons between studies. Here, 13 international experts propose specific guidelines designed to enhance the quality of research and publications on XC skiing, as well as on the biathlon and Nordic combined skiing. We consider biomechanical (kinematic, kinetic and neuromuscular) and physiological methodology (at the systemic and/or muscle level), providing recommendations for standardization/control of the experimental setup. We describe the types of measuring equipment and technology that are most suitable in this context. Moreover, we also deal with certain aspects of nomenclature of the classical and skating sub-techniques. In addition to enhancing the quality of studies on XC skiing, Nordic combined and biathlon, our guidelines should also be of value for sport scientists and coaches in other disciplines where physiological and/or biomechanical measurements are performed in the laboratory and/or outdoors.
\end{abstract}

Keywords Biomechanics $\cdot$ Measurement $\cdot$ Physiology $\cdot$ Reliability $\cdot$ Technology $\cdot$ Validity

\section{Introduction}

Approximately $30 \%$ of the medals awarded during the 2022 Winter Olympics in Beijing will go to athletes competing on "narrow skis" in the cross-country (XC) skiing, biathlon and Nordic combined disciplines, which involve

Hans-Christer Holmberg

integrativephysiobiomech@gmail.com

1 CeRiSM, University of Verona, Rovereto, Italy

2 Neuroscience, Biomedicine and Movement Science Department, University of Verona, Verona, Italy

3 Centre for Elite Sports Research, Department of Neuromedicine and Movement Science, Norwegian University of Science and Technology, Trondheim, Norway

4 Department of Sport Science and Kinesiology, University of Salzburg, Salzburg, Austria

5 Laboratory of Biomechanics, Faculty of Sport, University of Ljubljana, Ljubljana, Slovenia

6 Department of Sports Science and Clinical Biomechanics, University of Southern Denmark, Odense, Denmark complex biomechanics and considerable physiological challenges. The two styles of XC skiing, skating and classical, encompass five and four major sub-techniques, respectively, whereas biathletes and Nordic combined skiers utilize the skating technique exclusively [19, 25, 44, 42]. During a race,

7 Institute for Applied Training Science, Leizpig, Germany

8 Swiss Federal Institute of Sport, Magglingen, Switzerland

9 Department of Theory and Methods in Ski Sports, Russian State University of Physical Education, Sport, Youth and Tourism (SCOLIPE), Moscow, Russia

10 School of Kinesiology, University of British Columbia, Vancouver, B.C, Canada

11 Biomechanics Laboratory, Beijing Sport University, Beijing, China

12 Department of Biology of Physical Activity, University of Jyväskylä, Jyväskylä, Finland

13 Swedish Winter Sports Research Centre, Department of Health Sciences, Mid Sweden University, Östersund, Sweden 
skiers adapt to changes in the track by alternating between these various sub-techniques.

Research on XC skiing, Nordic combined and biathlon both in the laboratory and field, involves certain difficulties that may reduce the reliability and validity of the findings, as well as complicate comparisons between studies. Measurements outdoors on skis/roller skis often/in general exhibit higher external validity, but do not allow much of the sophisticated laboratory equipment available to be used and, moreover, may be complicated by adverse environmental conditions, such as poor weather or variations in snow and track conditions. Consequently, monitoring XC skiing indoors provides greater internal validity, accuracy and reliability.

Here, we discuss some of the methodological challenges associated with physiological and biomechanical research on $\mathrm{XC}$ skiing and propose guidelines designed to enhance the quality of research and publications on XC skiing, as well as on the biathlon and Nordic combined skiing.

\section{Participants}

All information reported about the participants (e.g., number, country, sex, age, stature and weight) should refer to the time-point at which the investigation began.

\section{Level}

The level of participants' performance should be indicated by their FIS (The Fédération Internationale de Ski)/IBU (International Biathlon Union) points or, for those without an international score, national points, if available, or finishing positions in national competitions. For junior and other sub-elite skiers who have not participated in many FIS/IBU races, their finishing positions in recent competitions and years of experience and/or biological and training age should be reported. In addition, the level at which the participants compete-e.g., Olympic, World Cup, international and/or national-as well as membership in any national team (senior or junior) should be mentioned.

Moreover, each subject's maximal or peak oxygen uptake $\left(\mathrm{VO}_{2 \max }\right.$ or $\left.\mathrm{VO}_{2 \text { peak }}\right)$, together with details of the protocol, including the skiing technique utilized, should be specified. Finally, all other information of potential relevance, such as previous training (e.g., hours/week, modes, etc.), as well as inclusion/exclusion criteria should be described.

\section{Provision of Nutrition, Fluids and Supplements During Measurements}

Nutrition, hydration and possible provision of supplements on the day of testing should be standardized and reported.
During the $24-48 \mathrm{~h}$ prior to testing, training should be standardized, light and reported. Skiers with recent injuries or who are ill and/or taking medication that could influence their performance should not participate. Any dietary restrictions should be described. The mode and intensity of warm-up should be standardized and reported, along with how familiar the athletes are with the experimental setup and the instructions they receive.

Performance by women could be affected by their menstrual stage [29] and/or consumption of hormones [11]. Although these effects appear to be trivial $[29,11]$, researchers should report this information about female participants [23].

\section{Designing the Set-Up for Measurements}

\section{Sub-techniques}

The classical and skating techniques consist of four and five major sub-techniques, respectively (Fig. 1, Table 1). Especially for skating, many different designations were introduced when this technique first came into use in the late 1980's. For maximal clarity, either the Gear or V system should be employed routinely, also indicating the other designation in parentheses [e.g., "The tests were performed using the G3 sub-technique (also known as V2)"]. In the case of skiing downhill, the designations are the same, regardless of which technique is employed (Fig. 1, Table 1).

\section{Information Concerning Measurements Outdoors that Should be Reported}

\section{General}

When collecting data during skiing on snow or roller-skiing outdoors, the conditions for all skiers should be similar and any variations documented. In this context, several parameters are of particular importance (Table 2). In addition, the conditions should be described in detail, to enable post-evaluation (and/or meta-analysis) of their potential influence on the results.

\section{The Track and Speed}

The minimal change in elevation for defining uphill and downhill sections (e.g., $10 \mathrm{~m}$ ) should be described, along with any special characteristics of the track and its preparation prior to testing (Table 2). Both speed and any variations therein should be reported. When the skiing speed is an independent variable, anything done to help the skier maintain the designated speed (e.g., light or acoustic signals, an accompanying vehicle) should be described. Additional information to be reported is listed in Table 2. 

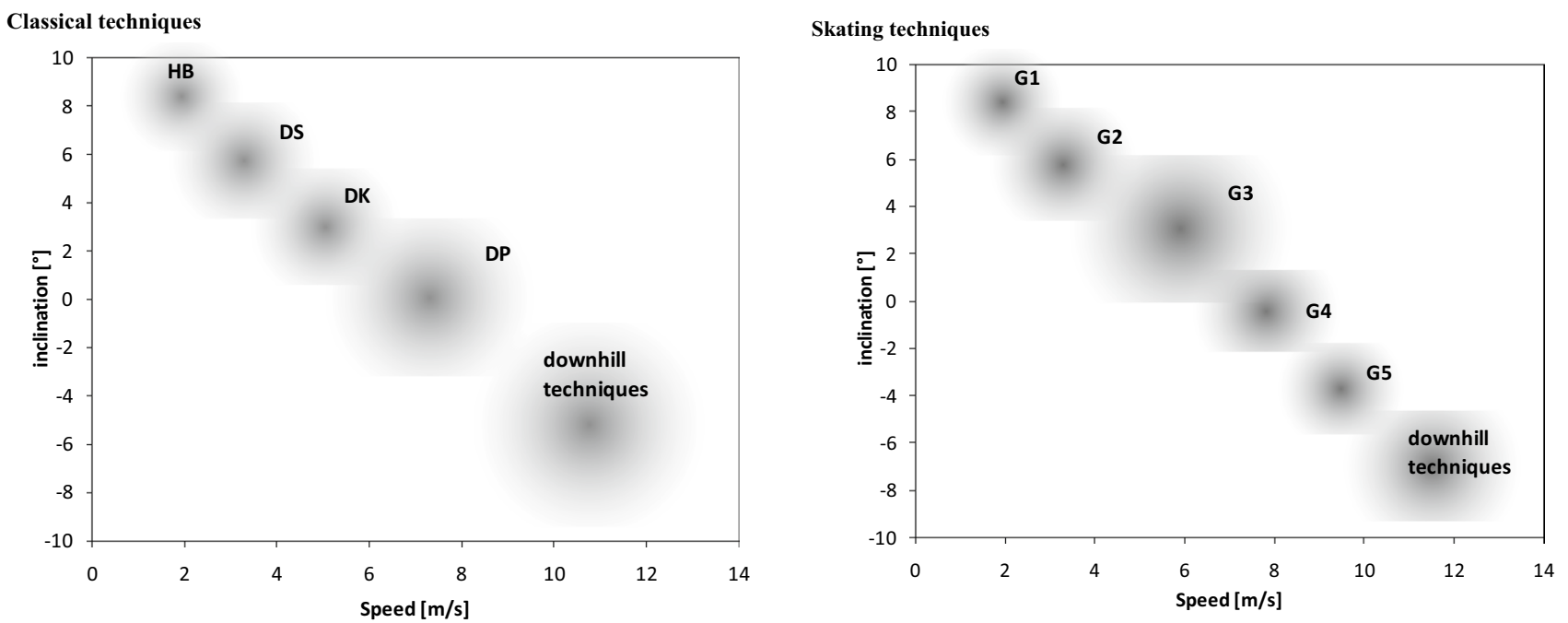

Fig. 1 Classical techniques and skating techniques (more details please see Table 1)

\section{Equipment}

Since interaction between the skis and snow (or roller-skis and ground) can exert a considerable impact on both biomechanical and physiological parameters, athletes should ideally utilize the same equipment (for roller skiing) or their own (on snow). If possible, all equipment should be prepared in a similar manner (ideally by the same technician, using the same ski wax and structure) and a gliding friction test performed. Alternatively, the change in the velocity of a skier (or with a load on the skis of comparable weight), as monitored either by speed traps, global navigation satellite systems (GNSS/GPS) or inertial measurement units (IMU) [51] when gliding passively can be determined. Either determination should be performed with the same skis to be utilized during the test.

When roller-skiing, the same pair should be used by all athletes or, alternatively, it should be confirmed that all roller-skis have similar characteristics, such as equal friction coefficients, prior to testing. Moreover, since temperature influences the rolling friction of wheels and ball bearings, roller skis should be warmed up for at least $10 \mathrm{~min}$ and/or kept in a warm box prior to testing $[1,2]$.

\section{Information Concerning Measurements Indoors}

Testing in the laboratory allows experimental conditions to be defined and controlled more precisely, as well as permitting the use of measuring systems/devices that may not be applicable outdoors. To ensure data of high validity and reliability, laboratory tests should be performed at a standardized, thermo-neutral temperature and the same time of the day, both of which should be reported.

\section{Treadmills and Ergometers}

The introduction of skiing treadmills in the mid-1990's enabled roller-skiing at a wide (and pre-defined) range of velocities $(0-40 \mathrm{~km} / \mathrm{h})$ and inclines $\left(-2-20^{\circ}\right)$, thereby allowing more ski-specific monitoring of both physiological and biomechanical parameters. Table 2 documents the information that should be reported when testing on a treadmill.

Testing and training with double poling (DP) ergometers (standing or sitting) require that certain factors be standardized and reported correctly to obtain valid and reliable results. In particular, the skier's position with respect to the ergometer (e.g., height of the pulley, horizontal distance, bench height) should be such that the angles of the joints and body closely resemble those during DP on snow and/or while roller skiing. Moreover, the level of resistance should be realistic, involving a poling motion that resembles that while performing DP on snow or while roller skiing as closely as possible, and, furthermore, should be standardized and reported, and the procedure for determining this resistance described.

\section{Biomechanical Measurements}

During recent decades, technological advancements that allow the collection of kinematic and kinetic data as well as information on neuromuscular factors have facilitated and improved biomechanical analysis of XC skiing.

The number of cycles that should be assessed to obtain reliable values for biomechanical parameters depends to a large extent on both the parameter and variability of the 


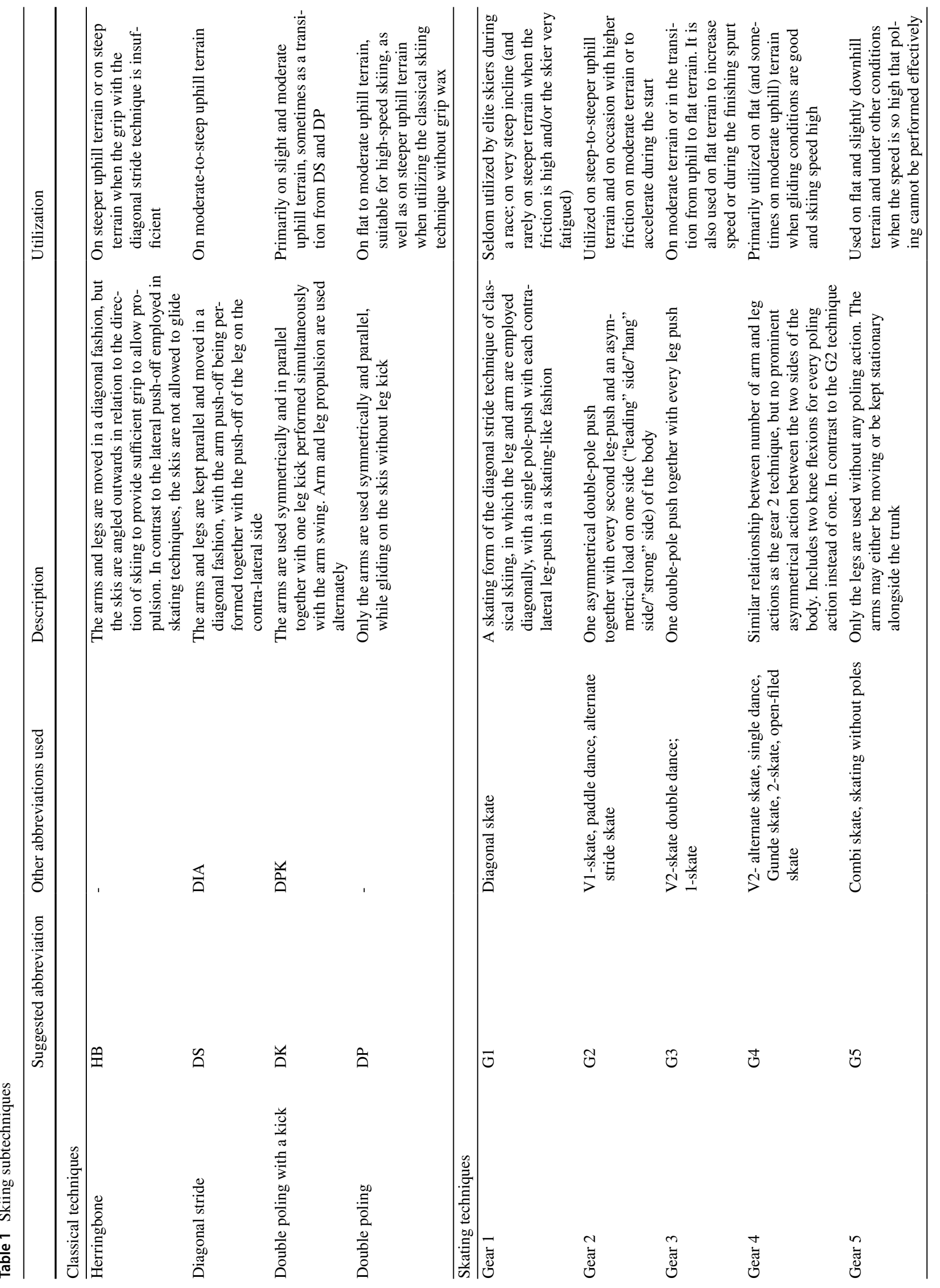




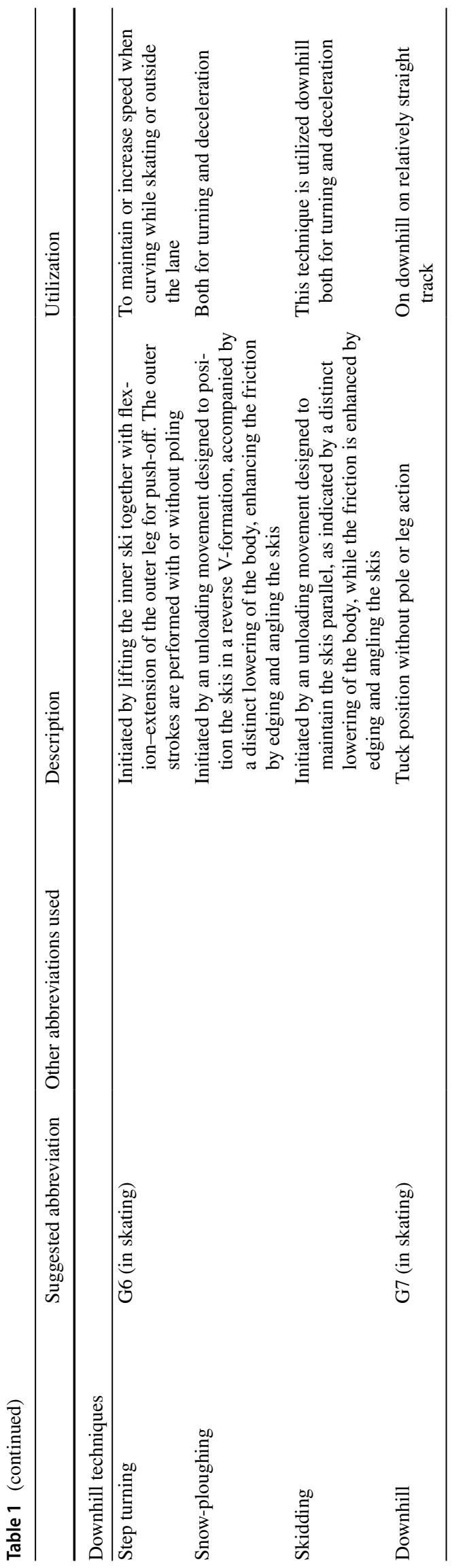

motion in question. In most cases, 15-30 cycles are considered both convenient and sufficient to provide accurate values. When making measurements in the field, the number of cycles that can be monitored depends on the technology employed. When video analysis is utilized, very few cycles are often monitored and on sections where skiing speed is high, monitoring 2 or 3 cycles is usually acceptable, although the more cycles monitored, the greater the reliability.

When analysing asymmetrical techniques (e.g., G2, G4, etc.), values for the strong and weak sides (the side on which poling is synchronized and not synchronized with ski thrust, respectively) should not be combined and this should be stated explicitly.

\section{Kinematics}

Kinematic analysis encompasses the characteristics of whole-body movements (e.g., cycle velocity, rate and/or length), as well as of isolated movements of individual joints (e.g., range of motion (RoM) and angular velocity) and body segments (e.g., velocities) during the performance of a specific XC skiing technique. Moreover, temporal data such as cycle and poling times can be determined. Kinematic measurements involve primarily various types of video recording with everything from a single, simple camera to more complex multi-camera opto-electronic motion capture systems (for monitoring displacement), as well as electrogoniometers (joint angles), IMU (acceleration, the orientation and angular velocity of body segments) and GPS/GNSS (position and velocity).

\section{Systems for Measuring Elapsed Times}

Elapsed time is widely used to quantify performance in numerous sports, including $\mathrm{XC}$ skiing. In addition to the total time elapsed, the time required to complete a lap or intermediate times provide further information about performance on sections of a course with different characteristics (e.g., flat or uphill terrain).

Among the many devices that measure time, singlebeamed photocells are most commonly utilized by both $\mathrm{XC}$ skiers themselves and researchers in this field. The reliability of these photocells is, however, highly dependent on the height at which the emitter and reflector are placed, which should be standardized and reported routinely. Moreover, the beams of light can be interrupted by different parts of the body or snowflakes. Therefore, when timing skiers at high velocity over short tracts, usage of dual-beamed (i.e., two photocells placed at different heights), split-beamed or post-processing photocells is recommended [16]. 
Table 2 Information to be reported concerning indoor and outdoor measurements

Skiing on snow Roller skiing Roller skiing on road/track on treadmill

\section{Course topography}

Altitude (min, max and average)

Length (in the case of the treadmill, the length available for skiing)

Width (min, max and average) (for the treadmill width available for skiing)

Inclination (min, max and average)

Total climb

Longest individual climb

Curves (number, direction and slopes)

A map (both in the horizontal and vertical directions) should be provided if possible (e.g., as an Appendix)

\section{Speed}

Speed (min, max and average)

Deviations from target speed

\section{Weather}

Air temperature (min, max and average)

Air humidity (min, max and average)

Weather conditions

Wind (speed and direction)

Use of a fan (in the laboratory)

Snow/ground temperature (min, max and average)

Snow conditions (type of (artificial or natural), age, latest grooming etc.) / ground condition (asphalt/concrete)/belt surface

\section{Time}

Duration of the experimental session

Time of day

Time of the year (also in relationship to the competitive season)

\section{Poles and skis/roller skis}

Type of ski boot/binding system (classical/skating)

Type of skis/roller skis (classical/skating)

Weight of the skis/roller skis

Ski preparation (grinding, grip wax, gliding wax for example Grip: Klister Red; Glide: Fluor paraffin + Fluor powder; Grinding: X45 (fine/straight structure)

Rolling/gliding friction (coefficient)

Type of wheels and presence/position of the ratcheted wheel

Information concerning pre-heating of the roller skis

Poles (material)

Pole length (fixed or adjustable)

Tip of the poles

$\begin{array}{lll}\mathrm{x} & \mathrm{x} & \mathrm{x} \\ \mathrm{x} & \mathrm{x} & \text { N.A. } \\ \mathrm{x} & \mathrm{x} & \mathrm{x} \\ \mathrm{x} & \mathrm{x} & \mathrm{x} \\ \mathrm{s} & \mathrm{s} & \text { N.A. } \\ \text { S } & \text { s } & \text { N.A. } \\ \text { N.A. } & \text { N.A. } & \text { s } \\ \mathrm{x} & \mathrm{x} & \text { N.A. } \\ \mathrm{x} & \mathrm{x} & \text { s }\end{array}$

$\mathrm{x}=$ mandatory, $\mathrm{s}=$ suggested, $\mathrm{N} . \mathrm{A} .=$ not applicable

Other devices, including video recording and various transponder systems, can be used for multiple determination of time elapsed over a long XC skiing course. In particular, the GNSS systems described below demonstrate high validity in this context [50]. Similarly, strong magnets placed at multiple positions along the course can be detected by inertial sensors worn by the athlete and provide section times [48].

A radar gun can monitor velocity over short and straight tracts at 50 or $100 \mathrm{~Hz}$, thereby allowing derivation of acceleration. The laser beam must be pointed along the direction of skiing, usually at the athlete's lower back. The velocity data obtained must be filtered, normally utilizing a cut-off frequency of $3 \mathrm{~Hz}$ [16]. 


\section{Video Cameras}

To date, 2-D video recordings have been most commonly employed for kinematic analysis. As long as certain conditions are met, the reliability of such data is comparable to that collected in the laboratory with more advanced optoelectronic motion capture systems [59].

To obtain temporal characteristics, such as cycle rate, poling time, ski recovery time, etc., good resolution is possible as long as the time-points at which the skis and pole touch the ground are clearly visible and the frame rate at least $50 \mathrm{~Hz}$. When more complex information is desired, e.g., displacement and angle, the setup and procedures must be more carefully controlled.

To minimize parallax errors in connection with 2-D video recording from the lateral side, the camera should be positioned alongside the section to be filmed, in the middle, pointing perpendicularly at the track and at a distance from the centre of the skiing lane at least double the length of the track itself. Calibration requires filming (in the plane of the skier's movements) a frame (or set of objects) whose dimension (or distance) is as large as possible and precisely known. With multiple skiing lanes, each lane should be calibrated individually [59].

Usage of two or more video cameras in combination, along with proper 3-D calibration, not only provides the 3-D coordinates of the points of interest but can also potentially minimize errors due to positioning of cameras or direction of filming. 3-D configurations can also provide kinematic data on skiers from directions other than lateral (e.g., from the front or behind). When the camera follows the skier, tracking software capable of managing the tilt/pan/zoom must be used.

Attachment of uniquely coloured patches/markers to landmark positions on the body and/or skiing equipment may help enhance the precision and speed of manual or semiautomatic digitalization of points of interest. Additional information to be reported is listed in Table 3 .

\section{Infrared Based-Motion Capture Systems}

Complex optoelectronic motion capture systems designed specifically for 3-D monitoring of human movement are less problematic and generally provide greater accuracy with higher sampling rates and less post-processing time, provided that calibration and processing are performed as recommended by the manufacturer. In some cases, special human body models including the equipment have to be created. In connection with skiing outdoors such systems can be disturbed by the reflection of sunlight on the snow, making their use in the field at present problematic at best and often impossible. However, the performance of motion capture systems (e.g., resolution, sunlight filters) is likely to be improved in the near future.

\section{Electrogoniometers}

Electrogoniometers allow direct measurement of elbow, hip, knee and ankle angular displacement during XC skiing [20, 26]. Again, provided that the recommended procedures for calibration are followed carefully, the reliability and validity of these devices are generally high and they are often used when other systems for motion analysis are unavailable or infeasible, e.g., when monitoring skiing outdoors. The type of device utilized (i.e., 1-D or 2-D, rigid or flexible), the application and calibration procedure should all be described. Flexible electrogoniometers are preferable, since these avoid errors due to misalignment of the centres of rotation of the joint and goniometer [54] and are in general more comfortable while skiing.

\section{Inertial measurements systems}

Wearable sensor technology has evolved dramatically during the past two decades and for 3-D-dimensional kinematic measurements of athletes outdoors, IMUs are replacing camera-based systems to a greater and greater extent [55].

Typically, an IMU consists of multiple sensors, accelerometers, gyroscopes, and/or magnetometers, each of which monitors in all three dimensions (providing a total of up to 9 axes). A single IMU is sufficient to determine cycle frequency and, in combination with, e.g., a GNSS that monitors instantaneous speed, also allows cycle length to be determined. For analysis of segmental kinematics (e.g., movements of the legs and/or arms, as well as of equipment such as the skis and/or poles), additional IMUs are required. When placed strategically on the skier, a single or two IMUs allow automatic identification, to a certain extent, of the major classical [47] and skating [40] subtechniques, respectively. When a chain of synchronized IMUs is attached to all major body segments (as, e.g., with the Xsens motion capture system) and combined with a GNSS, full-body 3-D kinematics can be monitored [49]. In this case, appropriate optimal sensor fusion algorithms, based primarily on Kalman filtering, must be applied [57]. When inertial sensors are used indoors, special precautions are required to avoid potential disturbance by magnetic fields. Information to be reported concerning IMU is listed in Table 4. 
Table 3 Information to be reported concerning video recording

Visual-2D Visual-3D Infrared motion capture system

\section{System}

Type of cameras (visual/IR)

Model, manufacturer

Number

Sampling frequency

Resolution

Fixed or pan/tilt zoom

Synchronization of video recordings (gen-lock, PPS from GPS, sound, trigger signal, body movement)

\section{Camera position}

Distance from the zone of measurement

Exact placement, including rotation and height

Dimension of the field of view

A schematic illustration of the recording procedure

The plane of the body (sagittal, frontal, transverse) being studied

\section{Calibration}

Number of calibration points

Dimension of the calibration object/points

The direction of the gravitational forces (yes/no, mode)

Orientation of the reference axes relative to the motion of the skier

\section{Point digitalization}

Type of markers, if any (active/passive, colours, dimensions)

\section{Number}

Position (s)

Schematic illustration of their positions

Anthropometric model employed

\section{Data processing}

Software employed

Manual or automatic recognition of markers

Gap filling (if any)

Description of the filtering (if any)

Estimated accuracy of positioning (when calculating other biomechanical parameters, their accuracy should also be reported)

Biomechanical calculations or any other manipulation of data should be explained in detail, prefer- $x$ ably with equations

$\mathrm{x}=$ mandatory, $\mathrm{s}=$ suggested, $\mathrm{N} . \mathrm{R} .=$ not required, $\mathrm{PPS}=$ pulse-per-second

\section{Global Navigation Satellite System (GNSS)}

A GNSS can be used for autonomous monitoring of the position [37] of a skier outdoors. The accuracy varies with the constellation of the satellites involved, their visibility (i.e., potential obstruction of signals by mountains, buildings, trees, etc.), and the degree of multipath, as well as other factors [56]. Therefore, simply referring to position accuracy determined in connection with a previous study performed under different conditions can be highly misleading.

In addition, this accuracy usually depends on the technique employed for positioning, as well as in some cases on the sampling frequency. A low-cost GNSS built into wristwatches or smartphones typically has an accuracy of $>2 \mathrm{~m}$, whereas top-quality GNSS provide real-time kinematic positioning with an accuracy of $<1$ and $<2 \mathrm{~cm}$ in the horizontal and vertical directions, respectively. For more advanced differential positioning, accuracy decreases as the distance between the rover and base station increases, particularly when this distance is large (e.g., $>5 \mathrm{~km}$, with $1 \mathrm{~mm}$ lower accuracy for each additional $\mathrm{km}$ ).

Most manufacturers of GNSS of higher quality supply information concerning the accuracy of each position surveyed, which provides for maximal reliability. Indeed, 
Table 4 Information concerning the IMUs employed that should be reported

\author{
The system itself \\ Manufacturer and model $\quad x$ \\ Data logging system (if this is not part of the IMU itself) and software involved $\quad x$ \\ Number of axes measured $\quad x$ \\ Number and accuracy of sensors $\quad x$ \\ Size and weight (sensors and data logging system combined) $\quad x$ \\ $\begin{array}{ll}\text { Communication between sensors } & \mathrm{x}\end{array}$ \\ Communication between sensors and data logging system $\quad x$ \\ Sampling frequency $\quad x$ \\ Estimated accuracy of parameters $\quad x$ \\ Synchronization with complementary systems, if applicable $\quad x$ \\ The measurement set-up and calibration \\ Positioning of the sensors and the data logging system on the athlete $\quad x$ \\ A schematic illustration/photograph of the positioning of sensors $\quad \mathrm{s}$ \\ The procedure used for and timing of calibration $\quad x$ \\ The duration of each measurement $s$ \\ Data processing \\ The software employed $\quad x$ \\ The coordinate system involved (e.g., global, somatic) s \\ Description of the filtering (if any) $\quad x$ \\ Description of how parameters not measured were determined: by biomechanical modelling or direct $\mathrm{x}$ \\ calculation by the system (preferably with provision of the equations employed) \\ Estimated accuracy of parameters

$\mathrm{x}=$ mandatory $; \mathrm{s}=$ suggested

if such information is available, it should certainly be provided (Table 5). When a low-cost system without information concerning position accuracy is utilized, this characteristic should be assessed in a preliminary study under appropriate conditions.

From a biomechanical perspective, interpreting GNSS data collected by an antenna located on the wrist (with a GNSS-enabled watch) as reflecting the position of the centre-of-mass (CoM) can lead to error. An antenna placed in the vicinity of the neck and shoulders is closer to the CoM while interfering minimally with satellite visibility [5, 45, 52]. Recently, Takeda and colleagues [52] demonstrated that a high-quality real-time kinematic GNSS can identify the technique being employed, the number of cycles performed and their duration, skiing speed, and skiing distance during a classical race with great precision.

\section{Kinetics}

Evaluation of pole force, as well as the force between the ski boots and skis helps explain the kinematic pattern observed. Moreover, valuable information on segmental kinematics can be obtained with an inversed dynamic approach [10].

The ground reaction forces associated with XC/roller skiing can be measured directly or estimated in several different ways - with external force platforms, pressure or sensor insoles in the footwear or force sensors integrated into the skis, bindings and/or poles-each with its own advantages and disadvantages. Although external force platforms allow high-frequency monitoring of 3-D force with great precision, these are expensive and, because of their limited length, even when several platforms are connected, only a few cycles of movement can be monitored. Moreover, they are only suitable for classical XC skiing, where the ski and pole forces are applied to the ground within a relatively narrow lateral space, without overlap [58]. In contrast, this approach is impractical for ski skating, where the contact of the skis and poles with the ground occurs across considerably broader and overlapping areas.

Alternatively, portable measuring systems can easily monitor consecutive cycles of any of the classical and skating techniques outdoors or on a treadmill. Among these, pressure insoles can be utilized to determine the normal force generated through the skis by the skier's legs, although with limited accuracy, since not all of the reaction force is exerted perpendicularly. Although the most common sampling frequency is $100 \mathrm{~Hz}$, some devices (e.g., the Pedar-X system) allow twice this frequency with a single insole.

For longer-term monitoring with higher accuracy, skis and poles instrumented with force sensors are probably most convenient. Since no such devices are commercially 
Table 5 Information concerning the GNSS employed that should be reported

\section{The system itself}

Manufacturer and model of the receiver (and antenna, if separate from the receiver)

Size and weight of the receiver and antenna

The satellites employed (e.g., United States GPS, Russian GLONASS, European Galileo, Chinese Beidou, other)

The procedure utilized for positioning, e.g., code-based or additional carrier-based (code-based DGNSS, Real Time Kinematics - RTK) or $\mathrm{x}$ post-processing with Precise Point Positioning (PPP)

The number of frequencies tracked, e.g., "single (L1)", "dual (L1/L2)"

\section{The measurement set-up}

With a single reference/base station, its distance to the area of measurement, with a network of reference stations (e.g., SBAS, GBAS and $\mathrm{x}$ providers (e.g., WAAS, EGNOS, QZSS)), the augmentation system employed

Sampling frequency

The accuracy of positioning

The number of satellites visible during the measurements

The geometric dilution of precision (GDOP) specifying the propagation of error

In the case of low-cost systems, whether speed is monitored on the basis of the Doppler effect

Positioning of the device on the athlete

A schematic illustration/photograph of this positioning

\section{Data processing}

The type of data filtering utilized, including crucial parameters such as the order of the filter, cut-off frequency and lag (if applicable)

The biomechanical modelling applied to calculate parameters not measured directly, preferably with provision of the equations utilized

$\mathrm{x}=$ mandatory $\mathrm{s}=$ suggested

available for skis at present and those for poles have come on the market only quite recently, researchers have developed their own systems [18, 22, 34]. When employing a system developed in-house, it is important to document its validity and accuracy, either by assessing these or providing appropriate references. The major limitation in this connection is the weight of the sensors, in most cases consisting of a cable-based system in combination with a data recorder, power supply, which influences the overall mass and mechanical properties of the skis and poles and should, therefore, be as light as possible and reported routinely.

The force measured by instrumented skis and poles is positioned in the 3-D reference system defined relative to the equipment. When information related to the direction of movement is required, determination of the orientation of the skis/roller skis $[14,22]$ and poles [46] allows the transformation of the force from the local coordinate system to global coordinates. Other information that should be reported is listed in Table 6.

\section{Neuromuscular Assessment}

With XC skiing, analysis of the temporal and frequency patterns in surface electromyography (sEMG) can provide useful information concerning the extent, duration and timing of muscular activation, as well as muscle fatigue. However, especially during dynamic contractions, various types of noise can interfere with sEMG signals, potentially leading to misinterpretation. Therefore, standard procedures for measuring and processing sEMG signals collected during dynamic muscle movements [31], must be adhered to and reported carefully (Table 7).

Standardized placement and fixation of the electrodes and cables to the skin (in a manner that does not impede movement or flexibility) and high-pass filtering of the sEMG signals with a $20 \mathrm{~Hz}$ cut-off [53] can help reduce such disturbance. By following guidelines, acceptable signals can be obtained even outdoors when, due to low temperatures, the skier needs to wear a suit over the electrodes.

Because of the key roles they play in producing propulsion, the superficial upper-arm, trunk, thigh and calf muscles are generally analysed. With the symmetrical techniques (G5, G3 and G1, double poling, diagonal stride or herringbone), analysis of one side of the body (generally the dominant side) is often sufficient, whereas analysis of both sides is preferable in the case of the asymmetrical techniques (G4, G2, double poling with kick). For comparison of the patterns of muscle activation on both sides of the body during the performance of the former techniques, bilateral placement of sEMG electrodes is required.

To reduce intra-subject variability, dynamic data are often normalized relative to the sEMG signal associated with maximal voluntary isometric contraction. However, if due to constrictions, unfamiliarity with the movement and/ or differences in the neural drive elicited, this contraction is not actually maximal, as is often the case, normalization may instead augment inter-subject variability [7]. On the 
Table 6 Information concerning the measurement of kinetic parameters that should be reported \begin{tabular}{ll} 
The system itself & $\mathrm{x}$ \\
Manufacturer and model (or if custom-designed) & $\mathrm{x}$ \\
Data logging system (if this is not part of the system itself) and software involved & $\mathrm{x}$ \\
Communication between sensors and data logging system & $\mathrm{x}$ \\
Size and weight (for devices worn on the skiers or skis and poles) & $\mathrm{x}$ \\
Size (for force platforms) & $\mathrm{x}$ \\
The type of sensors (force or pressure-based) & $\mathrm{x}$ \\
Number and orientation of axes measured & $\mathrm{s}$ \\
Full scale value for each axis & $\mathrm{x}$ \\
Sampling frequency & $\mathrm{x}$ \\
Estimated accuracy of parameters & $\mathrm{x}$ \\
Synchronization with complementary systems, if applicable & \\
The measurement set-up and calibration & $\mathrm{x}$ \\
The procedure used for and timing of calibration & $\mathrm{s}$ \\
The duration of each measurement & \\
Data processing & $\mathrm{x}$ \\
The software employed & $\mathrm{s}$ \\
The coordinate system involved (e.g., global, somatic) & $\mathrm{x}$ \\
Description of the type of data filtering (if any), including key parameters such as the order of the & filter, cut-off frequency and lag (if applicable) \\
Estimated accuracy of parameters & $\mathrm{x}$ \\
\hline
\end{tabular}

$\mathrm{x}=$ mandatory $; \mathrm{s}=$ suggested other hand, normalization on the basis of peak and/or mean sEMG signals during task-specific maximal contractions can reduce inter-subject variability, but does not allow detection of intra-individual changes or comparison of the activation of different muscles [7]. Alternatively, standardized normalization relative to maximal explosive isometric contractions, which involve maximal activation of specific muscles and elicit neural drives similar to those involved in task-specific contractions, may be appropriate [7].

Finally, sEMG signals can be employed to assess muscle fatigue utilizing algorithms developed specifically for application to dynamic contractions, such as the Cohen Class time-frequency approach [8]. Muscle fatigue can also be assessed on the basis of indices obtained by conventional Fast-Fourier Transformation [6], in which case the timecourse of muscle contractions during specific RoM must be taken into consideration. These types of analysis have also been applied to detect muscle fatigue during the repetitive movements involved in XC skiing [60, 61].

\section{Physiological Measurements}

The key determinants of performance commonly monitored during testing of XC skiers in the laboratory are a) $\mathrm{VO}_{2 \max }$ or $\mathrm{VO}_{2 \text { peak }}$, b) anaerobic energy contribution/power, c) indicators of the anaerobic threshold and d) work economy/ efficiency [42]. In addition, monitoring of physiological responses in the field provides more realistic insights, while analyses of muscle tissue help understand different aspects of the production and consumption of energy [19]. Here, we provide guidelines on how to standardize and report these various types of measurements.

\section{Respiratory Measurements}

In the case of $\mathrm{XC}$ skiers, respiratory variables (i.e., $\mathrm{VO}_{2}$, exhalation of carbon dioxide $\left(\mathrm{VCO}_{2}\right)$ and ventilation) can be assessed utilizing the Douglas Bag system (with a precision of $<2 \%$ ) or directly by online ergo-spirometry (with a precision of 3\%-5\%) (depending on the test conditions and quality of control). Optimally, the validity of these latter systems should be confirmed at least twice each year by comparing the values obtained during both submaximal and maximal exercise with those provided by the Douglas bag procedure (i.e., the golden standard). In addition, the device should be calibrated with a mechanical lung and the coefficient of variation of the difference in $\mathrm{VO}_{2}$ reported routinely.

Measurement of respiratory variables by online ergospirometry can be performed either with a mixing chamber or by the breath-by-breath procedure, each with strengths and limitations. Analysis of single breaths results in precision that is substantially lower and variation that is relatively large compared to averaging the $\mathrm{VO}_{2}$ during a period of, e.g., $30 \mathrm{~s}$ or a given number of breaths [28]. Similarly, with the mixing chamber, at least $30 \mathrm{~s}$ of monitoring is required, although longer periods provide a more accurate estimate and should be employed whenever possible. 
Table 7 Characteristics concerning the recording and processing of sEMG signals that should be reported

The muscles recorded

Number

Names

Location on which side(s) of the body

Surface electrodes

Type (monopolar, bipolar, array, matrix)

Material composition

Size and shape

Location (position, orientation, references for placement)

Inter-electrode distance

Surface and position of reference electrodes

\section{Preparation of the skin}

Cleaning procedures (shaving; abrasion; degreasing)

Skin impedance

\section{Minimization of potential electrical noise}

Precautions taken prior to measurement (isolation of external devices, examination of the sEMG signal frequencies) $\mathrm{s}$

Employment of software filters to reduce electrical noise due to other devices (types; cut-off frequencies; utilization of $\mathrm{dB} / \mathrm{octave}$ or $\mathrm{dB} / \quad \mathrm{x}$ decade for cut-off)

Utilization of software filters to reduce noise due to the thorax muscles (types; cut-off frequencies; utilization of dB/octave or dB/decade $\mathrm{s}$ for cut-off)

\section{The EMG device}

Mode of detection (monopolar, differential, double-differential, high-density EMG)

Sampling frequency (at least $1000 \mathrm{~Hz}$ )

Amplifier (type, gain)

Hardware filters (types; utilization of dB/octave or dB/decade for cut-off; bandwidth (5-500 Hz); signal-to-noise ratio)

A/D conversion card (model, frequency and number of bits employed for digitalization of time and amplitude)

Data transmission to storage device (analogue, wireless, optic fibre)

\section{Data processing}

Number of cycles analysed

Software filters (types; purposes; cut-off frequencies; utilization of $\mathrm{dB} /$ octave or $\mathrm{dB} /$ decade for cut-off)

Rectification procedure (full- or half-wave)

Enveloping procedures, if any (type of filter; cut-off frequencies; utilization of dB/octave or $\mathrm{dB} / \mathrm{dec}$ ade for cut-off; in the case of the moving window approach, time constant and mathematical operator)

Descriptors of the amplitude of the sEMG signals (RMs, ARV, EMG, calculated over an entire cycle or during specific sub-phases)

Procedure employed, if any, to detect onset, peak, offset and duration of muscle contraction (mathematical processing) $\quad \mathrm{x}$

Synchronization, if any, of sEMG data with other biomechanical measurements (type,

detection of a meaningful event in the cycle in relationship to the sEMG signals)

Computational procedures for analysis of muscle fatigue (if any)

\section{Normalization}

Type, including the nature of the contraction involved

$\mathrm{x}=$ mandatory, $\mathrm{s}=$ suggested

Previously, laboratory testing of respiratory variables has most often involved running, pole-walking or roller skiing on a treadmill or exercising on a cycling or doublepoling ergometer, which enables simultaneous precise online measurement of the external work rate. However, for this approach to be reliable the conditions employed must be controlled precisely, e.g., the treadmill speed and incline must be known exactly and the friction coefficients of roller skis measured. At the same time, technological developments and mobile measuring devices now allow metabolic 
parameters to be assessed in the field while skiing or roller skiing. In connection with such studies, the system utilized to determine respiratory parameters must have been validated for the prevailing conditions (i.e., temperature, humidity, altitude, etc.) and tolerate exposure to rain, snow, wind and altitude.

$\mathrm{VO}_{2 \max }$, the upper limit of aerobic capacity, is normally attained when exercising with a larger mass of muscle, such as when employing the diagonal skiing technique [21]. Thus, this is often the primary dependent variable when the status of and responses to training by endurance athletes are being characterized. In addition, a high $\mathrm{VO}_{2 \text { peak }}$ when performing the various sub-techniques in skiing, including a large upper-body capacity, is required for success in XC skiing [42]. Testing designed to determine $\mathrm{VO}_{2 \text { peak }}$ should be performed while roller skiing on a treadmill at relevant speeds and inclines or performing DP on an ergometer.

In connection with incremental or performance tests (e.g., with a time-trial) on a treadmill, the highest $\mathrm{VO}_{2}$ maintained for 30 or $60 \mathrm{~s}$ is usually defined as $\mathrm{VO}_{2 \text { max }} / \mathrm{VO}_{2 \text { peak }}$. Such a test can be considered acceptable when the plateau in $\mathrm{VO}_{2}$ is accompanied by a respiratory exchange ratio (RER) $>1.0$ and a blood level of lactate $[\mathrm{BLa}]>8 \mathrm{mmol} / \mathrm{L}$. The stages of a submaximal test should be at least 3 min long, but preferably $>4$ min, with recording of respiratory variables during the final minute of each bout. With progressive increments, it is possible that steady-state conditions can be reached in less than $3 \mathrm{~min}$, but this must be confirmed for each specific set of conditions. In either case, steady-state aerobic values are required to determine skiing economy/efficiency and identify the time-point at which respiratory variables or [BLa] begin to increase in a non-linear fashion (so-called break-points).

When indices of economy, efficiency or anaerobic thresholds are calculated on the basis of values obtained with submaximal exercise, these calculations should be described in detail and, moreover, we recommend that the values obtained at each stage be presented in the manuscript itself or as an appendix.

Immediately after both incremental, submaximal and performance tests, blood samples should be taken from the fingertip (or earlobe) for analysis of [BLa] and, in addition, the participants should be asked to rate their perceived exertion (RPE). In most cases, [BLa] is highest $1-3$ min after termination of maximal exercise.

In general, when reporting the values for metabolic parameters obtained by testing, it is imperative to describe in detail the system used for monitoring, the procedure for data processing, and the experimental set-up and protocols, as well as the preparation and status of the participants prior to testing (Table 8).

\section{Skiing Efficiency and Economy}

In connection with measurements of $\mathrm{VO}_{2}$, the aerobic metabolic rate can also be evaluated, by including the RER, an indicator of the relative (\%) utilization of substrate, and applying a conversion formula. Here, a prerequisite for obtaining a reliable RER is that the $\mathrm{CO}_{2}$ level in the inspired air be measured accurately and maintained constant (which is only possible in a large and well-ventilated setting). To allow attempts to reproduce findings, it is important to specify which conversion tables and/or equations were employed.

Gross efficiency (GE) is the most commonly employed measure of skiing efficiency and can be calculated from the following equation $[39,43]$ :

$G E[\%]=\frac{\text { work rate } \%[W]}{\text { metabolic rate } \%[W]} \times 100$

A major challenge in connection with the assessment of efficiency involves measuring the work performed accurately. The rate of work during roller skiing on a treadmill is usually estimated as the sum of the power exerted against gravity $(\mathrm{Pg})$ and against rolling friction (Pf). $\mathrm{Pg}$ is the product of mass ( $\mathrm{m}$; of both the body and equipment), gravitational acceleration $(\mathrm{g}), \sin (\alpha)$ and velocity $(\mathrm{v})$. Pf is calculated as the product of the friction coefficient $(\mu), \mathrm{m}$, $\mathrm{g}, \cos (\alpha)$ and treadmill velocity (v). Although the power exerted against gravity can be determined accurately, determination of the power required to overcome friction involves certain assumptions and simplifications, both in the case of skating [43] and classical skiing [38].

Researchers should always be aware that GE is dependent on the absolute rate of work and the sub-technique employed, as well as the combination of speed and incline employed [3, 41]. This potential problem can be minimized by comparing the athletes at similar work rates. In the case of flat roller skiing, this additional work approaches the zerowork paradox associated with running. Accordingly, the calculation of work rate should be described in detail and the challenges mentioned above kept in mind when investigating the impact of environmental conditions on the efficiency of skiing.

When the work rate cannot be measured accurately, skiing economy at defined submaximal speeds is commonly employed as an alternative. This economy is defined as the metabolic energy associated with skiing or running a defined distance and is obtained by dividing the metabolic rate by skiing speed [39].

\section{Heart Rate}

Heart rate (HR), a common measure of the relative intensity of endurance training, is often monitored during testing of 
Table 8 Information concerning physiological testing that should be reported

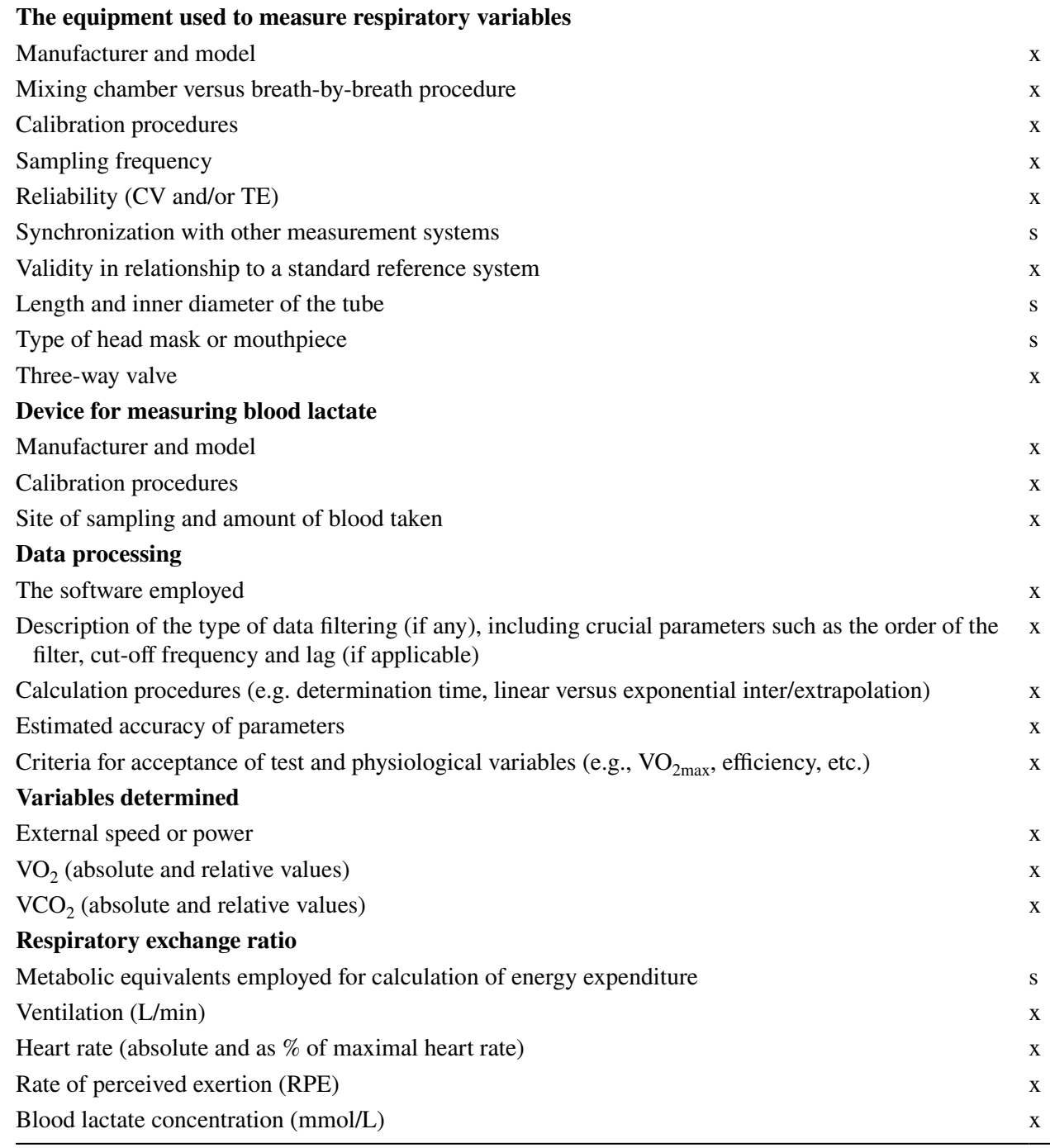

$\mathrm{x}=$ mandatory, $\mathrm{s}=$ suggested
$\mathrm{XC}$ skiers, both in the field and in the laboratory. However, interpretation of an individual's HR requires knowing his/ her maximal or peak HR, e.g., the highest HR maintained for $5 \mathrm{~s}$ during incremental exercise or 2-5-min bouts of maximal running or roller skiing/skiing. For submaximal testing, we recommend that HR be averaged over the same period of time as the corresponding gas exchange values (see further above). Over time, HR drifts to a greater extent than $\mathrm{VO}_{2}$, especially during exercise at higher intensities, and this rate should therefore be interpreted carefully in connection with longer test protocols [17]. With current technology, it is recommendable to measure heart rate with chest belts and not wrist sensors.

\section{Blood Lactate}

Blood lactate concentration [BLa] is one of the parameters commonly assessed during exercise testing. However, measurements of [BLa] must be carefully standardized and interpretations should be based on a detailed understanding of how lactate accumulates in the blood, as well as potential differences in the analytical procedure $[9,15]$. In the specific case of XC skiing, the response of [BLa] and calculation of the lactate threshold may differ between the different subtechniques, where the arms and legs are employed to different extents $[42,15]$.

The instrument used to measure [BLa] must be validated against the criterion/reference enzymatic determination. The devices in common use differ in precision and accuracy from this reference value by varying degrees, which must be taken into account. Moreover, the interpretation must take into consideration whether plasma or whole blood is being analysed, since [BLa] values for whole blood are, on average, $70 \%$ of the corresponding plasma values [13]. 


\section{Anaerobic Energy}

Performance in XC-skiing is also determined by the anaerobic energy contributed, i.e., anaerobic power or capacity. The most commonly utilized indicator of anaerobic capacity is the maximal accumulated oxygen deficit (MAOD) [30, 32]. Here, $\mathrm{VO}_{2}$ during repeated submaximal steady-state bouts of exercise are used to predict the $\mathrm{VO}_{2}$ demand at supra-maximal rates of work, allowing the accumulated $\mathrm{O}_{2}$ deficit to be calculated. However, in recent years determination of anaerobic power using the GE approach has been used as an alternative, especially since this method is less time-consuming [3, 4, 33]. In any case, details concerning the calculations performed, including the input variables, should be reported. At the same time, all present procedures for calculating anaerobic energy are relatively unreliable (typical error around 10\%) and the values obtained should be interpreted with caution [27].

\section{Evaluation of Muscle Tissue}

Important information regarding factors affecting the performance of and recovery from XC skiing can be obtained from direct measurements on muscle biopsies, including information on metabolic processes in exercising muscle, the influence of nutritional factors on muscle function, and muscle adaptations to strength and/or endurance exercise. Although biopsies have been analysed extensively by other researchers in exercise physiology, this approach has so far found only limited use in connection with XC skiing.

Although invasive, removal of a small piece of muscle tissue is relatively rapid and simple. In general, muscle biopsies are taken with three different techniques: (1) the needle biopsy, utilized in exercise science since the 1960's; (2) the open biopsy, involving cutting through the skin and into the muscle; and (3) the more up-to-date and minimally invasive micro-biopsy (or gun technique). Regardless of the procedure utilized, muscle biopsies can be taken quickly both before, during and after an intervention.

In this context, a major consideration is the muscle being sampled. In the case of classical XC skiing the $m$. vastus lateralis is usually analysed in connection with lowerbody exercise and the $m$. triceps brachii as an upper-body muscle $[12,35]$. Typically, the size of a needle biopsy is $100-150 \mathrm{mg}$, with less than a third as much material being obtained by micro-biopsy. The muscle sample can be homogenized, frozen in liquid nitrogen, or prepared in some other manner before analysis and/or storage.

One should always be aware that skeletal muscle is a heterogeneous tissue in which different fibre types exhibit unique contractile and metabolic properties. Thus, analysis of entire muscle biopsies, the approach most commonly employed, provides only average values. Analysis of single fibres supplies more detailed information but is also more time-consuming. Alternatively, appropriate histochemical preparation of cross-sections of muscle biopsies enables the area occupied by, distribution, and capillarization of individual types of fibres to be determined, along with their levels of different metabolites and proteins. However, analysis of the contractile properties of individual fibres provides much more precise insights that can be utilized to design more effective training and improve performance $[12,24,36]$.

\section{Conclusions}

Here, we present numerous recommendations concerning how to conduct research on XC skiing and the information that should be reported in connection with the publication of findings. We also deal with certain aspects of nomenclature regarding the classical and skating sub-techniques. The major biomechanical and physiological methodologies typically used in the laboratory and outdoor by researchers in this field are covered. When several different systems of measurement are utilized, it can be difficult to describe each in detail, especially when publishing in journals that restrict the word count. In such cases, although not ideal, other publications in which the methodology in question has been described can simply be referred to. In either case, the description of methodology should be sufficiently detailed to allow attempts to reproduce the findings. In short reports, the degree of detail should be proportional to its relevance to the primary aim of the investigation. Our purpose in providing these guidelines is to help improve the quality of investigations on XC skiing, Nordic combined and biathlon, and thereby promote more rapid and reliable advances in our understanding of this challenging sport.

Supplementary Information The online version of this article (https:// doi.org/10.1007/s42978-021-00112-6) contains supplementary material, which is available to authorized users.

Author Contributions BP, HC, ØS, MS, NØ and CZ drafted the first draft of this manuscript. TS, AS, TS, AL, CM, HL and OO read and commented on this first draft. All authors have read and approved the final version.

Funding Open access funding provided by Mid Sweden University.

Availability of Data and Material Not applicable.

\section{Compliance with Ethical Standards}

Conflict of interest On behalf of all authors, the corresponding author states that there is no conflict of interest. 
Open Access This article is licensed under a Creative Commons Attribution 4.0 International License, which permits use, sharing, adaptation, distribution and reproduction in any medium or format, as long as you give appropriate credit to the original author(s) and the source, provide a link to the Creative Commons licence, and indicate if changes were made. The images or other third party material in this article are included in the article's Creative Commons licence, unless indicated otherwise in a credit line to the material. If material is not included in the article's Creative Commons licence and your intended use is not permitted by statutory regulation or exceeds the permitted use, you will need to obtain permission directly from the copyright holder. To view a copy of this licence, visit http://creativecommons.org/licenses/by/4.0/.

\section{References}

1. Ainegren M, Carlsson P, Tinnsten M. Rolling resistance for treadmill roller skiing. Sports Eng. 2008;11:23-9.

2. Ainegren M, Carlsson P, Tinnsten M. Roller ski rolling resistance and its effects on elite athletes' performance. Sports Eng. 2009;11(3):143-57.

3. Andersson E, Björklund G, Holmberg HC, Ørtenblad N. Energy system contributions and determinants of performance in sprint cross-country skiing. Scand J Med Sci Sports. 2016;27(4):385-98.

4. Andersson EN, Noordhof DA, Lögdal N. The anaerobic capacity of cross-country skiers: the effect of computational method and skiing sub-technique. Front Sports Act Living. 2020;2:37.

5. Andersson E, Supej M, Sandbakk O, Sperlich B, Stöggl T, Holmberg HC. Analysis of sprint cross-country skiing using a differential global navigation satellite system. Eur J Appl Physiol. 2010;110(3):585-95.

6. Arabadzhiev TI, Dimitrov GV, Dimitrova NA. Simulation analysis of the performance of a novel high sensitive spectral index for quantifying M-wave changes during fatigue. J Electromyogr Kinesiol. 2005;15(2):149-58.

7. Ball N, Scurr J. Electromyography normalization methods for high-velocity muscle actions: review and recommendations. J Appl Biomech. 2013;29(5):600-8.

8. Bonato P, Roy SH, Knaflitz M, De Luca CJ. Time-frequency parameters of the surface myoelectric signal for assessing muscle fatigue during cyclic dynamic contractions. IEEE Transcriptions on Biomedical Engineering. 2001;48(7):745-53.

9. Brooks GA, Gladden LB. The metabolic systems: anaerobic metabolism (glycolytic and phosphagen). In: Society AP, editor. Exercise Physiology. Tipton: Oxford University Press; 2003. p. 332-60.

10. Danielsen J, Sandbakk $\varnothing$, McGhie D, Ettema G. Mechanical energetics and dynamics of uphill double-poling on rollerskis at different incline-speed combinations. PLoS ONE. 2019;14(2):e0212500.

11. Elliott-Sale KJ, McNulty KL, Ansdell P, Goodall S, Hicks KM, Thomas K, Swinton PA, Dolan E. The effects of oral contraceptives on exercise performance in women: a systematic review and meta-analysis. Sports Med. 2019;50(10):1785-812.

12. Gejl KD, Ørtenblad N, Andersson E, Plomgaard P, Holmberg HC, Nielsen J. Local depletion of glycogen with supramaximal exercise in human skeletal muscle fibres. J Physiol. 2017;595(9):2809-21.

13. Goodwin ML, Harris JE, Hernandez A, Gladden LB. Blood lactate measurements and analysis during exercise: a guide for clinicians. J Diabetes Sci Technol. 2007;1(4):558-69.

14. Göpfert C, Lindinger SJ, Ohtonen O, Rapp W, Müller E, Linnamo $\mathrm{V}$. The effect of swinging the arms on muscle activation and production of leg force during ski skating at different skiing speeds. Hum Mov Sci. 2016;47:209-19.
15. Van Hall G, Jensen-Urstad M, Rosdahl H, Holmberg HC, Saltin B, Calbet JA. Leg and arm lactate and substrate kinetics during exercise. Am J Physiol Endocrinol Metab. 2003;284(1):E193-205.

16. Haugen T, Buchheit M. Sprint running performance monitoring: methodological and practical considerations. Sports Med. 2016;46(5):641-56.

17. Haugnes $P$, Kocbach J, Luchsinger H, Ettema G, Sandbakk $\varnothing$. The interval-based physiological and mechanical demands of cross-country ski training. Int J Sports Physiol Perform. 2019;14(10):1371-7.

18. Hladnik J, Supej M, Jerman B. Force measurement system for roller-ski skating. Tehnicki Vjesnik-Technical Gazette. 2018;25(5):1291-7.

19. Holmberg HC. The elite cross-country skier provides unique insights into human exercise physiology. Scand J Med Sci Sports. 2015;25(Suppl 4):100-9.

20. Holmberg HC, Lindinger S, Stöggl T, Eitzlmair E, Müller E. Biomechanical analysis of double poling in elite cross-country skiers. Med Sci Sports Exerc. 2005;37(5):807-18.

21. Holmberg HC, Rosdahl H, Svedenhag J. Lung function, arterial saturation and oxygen uptake in elite cross country skiers: influence of exercise mode. Scand J Med Sci Sports. 2007;17(4):437-44.

22. Hoset M, Rognstad AB, Rølvåg T, Ettema G, Sandbakk Ø. Construction of an instrumented roller ski and validation of three-dimensional forces in the skating technique. Sports Eng. 2014; 17:23-32.

23. Janse DEJX, Thompson B, Han A. Methodological recommendations for menstrual cycle research in sports and exercise. Med Sci Sports Exerc. 2019;51(12):2610-7.

24. Kent JA, Ørtenblad N, Hogan MC, Poole DC, Musch TI. No muscle is an island: integrative perspectives on muscle fatigue. Med Sci Sports Exerc. 2016;48(11):2281-93.

25. Laaksonen MS, Jonsson M, Holmberg HC. The olympic biathlon - recent advances and perspectives after pyeongchang. Front Physiol. 2018;9:796.

26. Lindinger SJ, Holmberg HC. How do elite cross-country skiers adapt to different double poling frequencies at low to high speeds? Eur J Appl Physiol. 2011;111(6):1103-19.

27. Losnegard T. Energy system contribution during competitive cross-country skiing. Eur J Appl Physiol. 2019;119(8):1675-90.

28. Martin-Rincon M, Calbet JA. Progress update and challenges on VO2max testing and interpretation. Front Physiol. 2020. https:// doi.org/10.3389/fphys.2020.01070.

29. McNulty KL, Elliott-Sale KJ, Dolan E, Swinton PA, Ansdell P, Goodall S, Thomas K, Hicks KM. The effects of menstrual cycle phase on exercise performance in eumenorrheic women: a systematic review and meta-analysis. Sports Med. 2020;50(10):1813-27.

30. Medbø JI, Mohn AC, Tabata I, Bahr R, Vaage O, Sejersted OM. Anaerobic capacity determined by maximal accumulated $\mathrm{O} 2$ deficit. J Appl Physiol. 1988;64(1):50-60.

31. Merletti R, Di Torino P. Standards for reporting EMG data. J Electromyogr Kinesiol. 1999;9(1):3-4.

32. Noordhof DA, de Koning JJ, Foster C. The maximal accumulated oxygen deficit method: a valid and reliable measure of anaerobic capacity? Sports Med. 2010;40(4):285-302.

33. Noordhof DA, Skiba PF, de Koning JJ. Determining anaerobic capacity in sporting activities. Int J Sports Physiol Perform. 2013;8(5):475-82.

34. Ohtonen O, Lindinger S, Lemmettyla T, Seppala S, Linnamo V. Validation of portable 2D force binding systems for cross-country skiing. Sports Eng. 2013;16:281-96. 
35. Ørtenblad N, Nielsen J, Boushel R, Söderlund K, Saltin B, Holmberg HC. The muscle fiber profiles, mitochondrial content, and enzyme activities of the exceptionally well-trained arm and leg muscles of elite cross-country skiers. Front Physiol. 2018;9:1031.

36. Ørtenblad N, Nielsen J, Saltin B, Holmberg HC. Role of glycogen availability in sarcoplasmic reticulum $\mathrm{Ca} 2+$ kinetics in human skeletal muscle. J Physiol. 2011;589(Pt 3):711-25.

37. Parkinson BW, Spilker JJ. Global positioning system: theory and application, vol. 1. Washington, DC: American Institute of Aeronautics and Astronautics; 1996.

38. Pellegrini B, Zoppirolli C, Bortolan L, Zamparo P, Schena F. Gait models and mechanical energy in three cross-country skiing techniques. J Exp Biol. 2014;217(Pt 21):3910-8.

39. di Prampero PE. The energy cost of human locomotion on land and in water. Int J Sports Med. 1986;7(2):55-72.

40. Rindal OMH, Seeberg TM, Tjønnås J, Haugnes P, Sandbakk Ø. Automatic classification of sub-techniques in classical cross-country skiing using a machine learning algorithm on micro-sensor data. Sensors (Basel). 2018;18(1):75.

41. Sandbakk $\varnothing$, Ettema G, Holmberg HC. The influence of incline and speed on work rate, gross efficiency and kinematics of roller ski skating. Eur J Appl Physiol. 2012;112(8):2829-38.

42. Sandbakk $\varnothing$, Holmberg HC. Physiological capacity and training routines of elite cross-country skiers: approaching the upper limits of human endurance. Int J Sports Physiol Perform. 2017;12(8):1003-11.

43. Sandbakk $\varnothing$, Holmberg HC, Leirdal S, Ettema G. Metabolic rate and gross efficiency at high work rates in world class and national level sprint skiers. Eur J Appl Physiol. 2010;109(3):473-81.

44. Sandbakk $\varnothing$, Rasdal V, Braten S, Moen F, Ettema G. How do world-class nordic combined athletes differ from specialized cross-country skiers and ski jumpers in sport-specific capacity and training characteristics? Int J Sports Physiol Perform. 2016;11(7):899-906.

45. Sandbakk $\emptyset$, Sandbakk SB, Supej M, Holmberg HC. The velocity and energy profiles of elite cross-country skiers executing downhill turns with different radii. Int J Sports Physiol Perform. 2014;9(1):41-7.

46. Stöggl T, Holmberg HC. Force interaction and 3D pole movement in double poling. Scand J Med Sci Sports. 2011;21(6):E393-404.

47. Stöggl T, Holst A, Jonasson A, Andersson E, Wunsch T, Norström C, Holmberg HC. Automatic classification of the sub-techniques (gears) used in cross-country ski skating employing a mobile phone. Sensors (Basel). 2014;14(11):20589-601.

48. Stöggl T, Ohtonen O, Takeda M, Miyamoto N, Snyder C, Lemmettylä T, Linnamo V, Lindinger SJ. Comparison of exclusive double poling to classic techniques of cross-country skiing. Med Sci Sports Exerc. 2019;51(4):760-72.

49. Supej M. 3D measurements of alpine skiing with an inertial sensor motion capture suit and GNSS RTK system. J Sports Sci. 2010;28(7):759-69.
50. Supej M, Holmberg HC. A new time measurement method using a high-end global navigation satellite system to analyze alpine skiing. Res Q Exerc Sport. 2011;82(3):400-11.

51. Swarén M, Karlöf L, Holmberg H-C, Eriksson A. Validation of test setup to evaluate glide performance in skis. Sports Technol. 2014;7(1-2):89-97.

52. Takeda M, Miyamoto N, Endo T, Ohtonen O, Lindinger S, Linnamo V, Stöggl T. Cross-country skiing analysis and ski technique detection by high-precision kinematic global navigation satellite system. Sensors (Basel). 2019;19(22):4947.

53. Tankisi H, Burke D, Cui L, de Carvalho M, Kuwabara S, Nandedkar SD, Rutkove S, Stålberg E, van Putten MJAM, FuglsangFrederiksen A. Standards of instrumentation of EMG. Clin Neurophysiol. 2020;131(1):243-58.

54. Tesio L, Monzani M, Gatti R, Franchignoni F. Flexible electrogoniometers: kinesiological advantages with respect to potentiometric goniometers. Clin Biomech (Bristol, Avon). 1995;10(5):275-7.

55. Teufl W, Miezal M, Taetz B, Fröhlich M, Bleser G. Validity of inertial sensor based 3D joint kinematics of static and dynamic sport and physiotherapy specific movements. PLoS ONE. 2019;14(2):e0213064.

56. Teunissen P, Montenbruck O. Handbook of Global Navigation Satellite Systems. Springer: Springer Handbooks; 2017.

57. Tognetti A, Lorussi F, Carbonaro N, de Rossi D. Wearable goniometer and accelerometer sensory fusion for knee joint angle measurement in daily life. Sensors (Basel). 2015;15(11):28435-55.

58. Vähäsöyrinki P, Komi PV, Seppala S, Ishikawa M, Kolehmainen V, Salmi JA, Linnamo V. Effect of skiing speed on ski and pole forces in cross-country skiing. Med Sci Sports Exerc. 2008;40(6):1111-6.

59. Zoppirolli C, Bortolan L, Stella F, Boccia G, Holmberg H-C, Schena F, Pellegrini B. Following a long-distance classical race the whole-body kinematics of double poling by elite cross-country skiers are altered. Front Physiol. 2018;9:978.

60. Zoppirolli C, Pellegrini B, Bortolan L, Schena F. Effects of short-term fatigue on biomechanical and physiological aspects of double poling in high-level cross-country skiers. Hum Mov Scie. 2016;47:88-97.

61. Zory R, Barberis M, Rouard A. Kinematics of sprint cross-country skiing. Acta Bioeng Biomech. 2005;7(2):87-96.

Publisher's Note Springer Nature remains neutral with regard to jurisdictional claims in published maps and institutional affiliations. 